\title{
A APLICAÇÃO DE ADESIVO DE CIANOACRILATO POR HISTEROSCOPIA E OS TESTES DE PERVIEDADE NA ESTERILIZAÇÃO TUBÁRIA DE OVELHAS
}

\author{
PATENCY TESTS ON SHEEP UTERINE TUBE STERILIZATION AFTER HYSTEROSCOPIC \\ CYANOACRYLATE USAGE
}

\author{
Sergio Bigolin'; Djalma José Fagundes, TCBC-SP²; Henri Chaplin Rivoire, TCBC-RS³; \\ Ricardo Santos Simões ${ }^{4}$; Anna Tereza Negrini Fagundes ${ }^{5}$; Manuel de Jesus Simões ${ }^{6}$
}

\begin{abstract}
RESUMO: Objetivo: avaliar, por meio de testes de prenhez e de perviedade, a eficácia da obstrução tubária de ovelhas com adesivo de cianoacrilato aplicado por via histeroscópica. Método: 12 ovelhas adultas, com uma gestação anterior, foram distribuídas em três grupos: G-PO (n=3) submetidas à laqueadura tubária tipo Pomeroy; G-SH (n=3) aplicação de solução salina no istmo tubário por histeroscopia, e G-AD $(\mathrm{n}=6)$ aplicação de $0,5 \mathrm{~mL}$ de $\mathrm{n}$-butil-2-cianoacrilato no interior do istmo tubário, por histeroscopia. Depois de acasaladas por 90 dias com machos de comprovada fertilidade, as ovelhas não prenhas foram submetidas à laparotomia, retiradas as tubas uterinas, medidos os seus diâmetros e preparadas para testes de perviedade pela aplicação de azul de metileno e de pressão hidráulica (80mmHg). Resultados: As ovelhas de G-PO e G-AD não ficaram prenhas (0\%), em contraste com as SH (100\%). O GPO apresentou maior concentração de aderências $(66,6 \%)$ e de granulomas $(100 \%)$ em relação ao grupo AD (0\%); o adesivo manteve-se íntegro no lúmen tubário. O diâmetro da tuba uterina em G-AD $(0,650 \pm 0,16 \mathrm{~cm})$ foi significantemente maior do que o das tubas em G-PO $\left(0,195 \pm 0,04^{*} \mathrm{~cm}\right)(\mathrm{p}=0,01)$. Os testes de perviedade de pressão e corante foram negativos para todo o grupo PO e somente um animal em AD mostrou-se positivo para o teste de pressão. Conclusão: a aplicação histeroscópica de cianoacrilato no lúmen tubário de ovelhas impediu eficientemente a fecundação, constituindo procedimento eficaz e de boas perspectivas para aplicação futura em humanos (Rev. Col. Bras. Cir. 2008; 35(1): 023-027).
\end{abstract}

Descritores: Tubas uterinas; Histeroscopia; Esterilização reprodutiva; Adesivos teciduais; Ovinos.

\section{INTRODUÇÃO}

A esterilização cirúrgica representa um importante meio de contracepção em todo o mundo, sendo que a laqueadura tubária é o método anticoncepcional mais usado pelas mulheres brasileiras ${ }^{1}$. Dados da última Pesquisa Nacional sobre Demografia e Saúde (PNDS) de 1996 mostraram uma prevalência de $40 \%$ de esterilização para mulheres unidas de 15 a 49 anos sendo que, dependendo da região considerada, $50 \%$ a $70 \%$ das laqueaduras são realizadas durante a cesariana, muitas vezes indicada somente para atender ao desejo da esterilização ${ }^{2}$. Dados de outra pesquisa nacional referem que a esterilização cirúrgica é realizada durante ou imediatamente após o último parto (88\%), sendo que a maioria delas (77\%) por meio de uma cesariana ${ }^{3}$.

A laqueadura tubária é um procedimento legal (Lei Federal 9.263/1996), autorizado pelo Sistema Único de Saúde (Portaria 114/1997) 3 . O aumento do número de procedimentos de esterilização feminina (293 registros em 1996 para 15.370 em 2001), os estados e municípios terem limites anuais (numéricos e financeiros) de internação pelo SUS e os leitos para esterilização competirem com outras necessidades de saúde são fatores relevantes no tempo de espera para uma esterilização $\mathrm{o}^{2,3}$.

Portanto, para atender as expectativas individuais e sociais de planejamento familiar há, entre outras, a necessidade de desenvolver procedimentos operatórios de simples e rápida execução em regime ambulatorial ou em hospital-dia. Nestas condições, a histeroscopia para obstrução tubária por via transcervical é uma opção promissora. Atualmente destaca-se um procedimento $\left(\right.$ Essure $\left.^{\circledR}\right)$, que por meio da histeroscopia, possibilita a inserção no lúmen tubário de microdispositivo sintético expansível que provoca uma reação inflamatória local intensa e consequentemente à obstrução tubária. Embora de custo menor do que a laqueadura convencional ainda assim é oneroso para aplicação em larga escala devido à necessidade de equipamentos especiais para a aplicação e a aquisição do próprio dispositivo ${ }^{4}$.

\footnotetext{
1. Professor Assistente da Disciplina de Embriologia da Universidade Regional Integrada do Alto Uruguai e Missões-Campus Erechim - Rio Grande do Sul (RS)

2. Professor Adjunto da Disciplina de Técnica Operatória e Cirurgia Experimental do Departamento de Cirurgia da Universidade Federal de São Paulo - São Paulo (SP).

3. Professor Adjunto da Disciplina de Técnica Cirúrgica e Chefe do Departamento de Cirurgia da Fundação Universidade do Rio Grande - Rio Grande do Sul (RS).

4. Médico Residente dos Departamentos de Ginecologia e Obstetrícia da Universidade Federal de São Paulo - São Paulo (SP).

5. Acadêmica do $5^{\circ}$. Ano de Medicina da Universidade de Santo Amaro, São Paulo (SP).

6. Professor Livre-Docente da Disciplina de Histologia do Departamento de Morfologia da Universidade Federal de São Paulo - UNIFESP - EPM
} Recebido em 08/08/2007

Aceito para publicação em 10/10/2007

Conflito de interesses: nenhum

Fonte de financiamento: nenhuma

Trabalho realizado no Departamento de Cirurgia Experimental da Universidade Federal de São Paulo - UNIFESP - EPM. 
$\mathrm{O}$ adesivo de cianoacrilato tem sido usado em diversos tecidos e órgãos ${ }^{5,6}$, mas há escassez de trabalhos específicos voltados para a esterilização tubária na literatura ${ }^{7-10}$. Autores nacionais desenvolveram trabalhos experimentais de obstrução tubária em coelhas com uso de adesivos ${ }^{11,12}$ e demonstraram a adequação do cianoacrilato. Embora a coelha seja o animal de escolha na literatura para estudos de esterilização, notou-se a necessidade de ampliar o estudo para um modelo de animal que tivesse uma tuba uterina com dimensões e estrutura similar a de seres humanos, como as ovelhas $^{13}$.

Deste modo pareceu-nos pertinente estudar a associação da aplicação tubária do n-butil-2-cianoacrilato com auxílio da histeroscopia. Além de ser possível aplicar também em regime ambulatorial e sem anestesia o custo do adesivo seria menor que os microdispositivos em estudo referidos pela literatura.

O objetivo deste trabalho é avaliar a eficácia do método de esterilização, com a oclusão com n-butil 2-cianoacrilato, aplicado por via transcervical, em tubas uterinas de ovelhas.

\section{MÉTODOS}

A pesquisa foi aprovada pela Comissão de Ética do Hospital São Paulo - Universidade Federal de São Paulo - Escola Paulista de Medicina (CEP n ${ }^{\circ}$. 1352/04) e ratificada a aprovação pelo Comitê de Bioética em Pesquisa da Universidade Regional Integrada do Alto Uruguai e Missões - Campus Erechim (nº.160-1/PIA/04).

Doze ovelhas (Ovis aries) da raça Texel, com peso médio de $55 \mathrm{Kg}$ e idade de três anos, todas férteis com uma comprovada prenhez e prole anterior, foram distribuídas aleatoriamente em três grupos, a saber: G-PO (Pomeroy) com três animais e seis tubas uterinas; G-SH (Sham) com três animais e seis tubas uterinas e, G-AD (Adesivo) com seis animais e doze tubas uterinas.

Os ovinos foram anestesiados pela aplicação intramuscular da associação de xilazina $\left(0,2 \mathrm{mg} \cdot \mathrm{Kg}^{-1}\right)$ e tiletamina/ zolazepan (3mg. $\left.\mathrm{Kg}^{-1}\right)$. Todos os animais receberam ampicilina sódica na dose de $20 \mathrm{mg} . \mathrm{Kg}^{-1}$ como antibioticoprofilaxia.

As ovelhas do Grupo PO foram submetidas a procedimento operatório para esterilização segundo o método proposto por Pomeroy. Em condições de assepsia e anti-sepsia foi realizada incisão mediana infra-umbilical $(10 \mathrm{~cm})$ com exposição da cavidade abdominal e identificação dos istmos das tubas uterinas direito e esquerdo. Realizada a dupla ligadura com categute simples 000 e retirado $1 \mathrm{~cm}$ do segmento de cada tuba entre as duas ligaduras. Síntese da parede abdominal em três planos, o peritônio parietal com categute simples 000 em sutura contínua, a aponeurose em sutura com pontos separados e a pele com pontos simples com fio 4-0 de poliglactina 910 .

As ovelhas do Grupo SH e do Grupo AD foram colocadas em decúbito ventral, e com espéculo vaginal identificado o colo do útero. Pinçou-se e tracionou-se o colo do útero para expor o óstio do útero. Com óptica de $4 \mathrm{~mm} / 30^{\circ}$ em camisa operatória ligada ao "histeroflator" e fonte de luz Xenon-300 Watts, foi iniciado o procedimento videohisteroscópico. A distensão do colo do útero e do corpo do útero foi realizada com $\mathrm{CO}_{2}$ inflado à $80 \mathrm{mmHg}$ e $50 \mathrm{~mL} /$ minuto. Identificaram-se os cornos uterinos direito e esquerdo com os respectivos óstios uterinos das tubas. Pelo canal de trabalho da camisa operatória, passou-se um cateter ureteral $5 \mathrm{Fr}$ que possuía marcas a cada $1 \mathrm{~cm}$ de extensão, facilitando o seu correto e padronizado posicionamento na tuba uterina para a instilação de $0,5 \mathrm{~mL}$ de solução fisiológica $(\mathrm{G}-\mathrm{SH})$ ou de $0,5 \mathrm{~mL}$ de adesivo (G-AD) no istmo da tuba uterina.

Após quatorze dias dos procedimentos operatórios todas as ovelhas foram mantidas permanentemente até o nonagésimo dia em regime de acasalamento com machos de comprovada fertilidade (que já foram acasalados com outras ovelhas). Após 90 dias todas as ovelhas foram submetidas ao exame físico para diagnóstico de gestação.

Nas ovelhas com avaliação negativa para gestação procedeu-se à eutanásia, pelo método físico do atordoamento (martelo pneumático) seguida da secção das carótidas, e foram submetidas à laparotomia para avaliação macroscopia da presença ou ausência de granulomas e aderências.

Em seguida, foram retirados o útero, tubas uterinas e ovários por ressecção em monobloco sendo as tubas uterinas submetidas a dois testes in vitro para a perviedade tubária e medição do diâmetro transversal na região imediatamente anterior ao implante do adesivo ou da secção, assim como o comprimento do implante. Para tanto foi utilizado paquímetro digital Kingtools-150mm com precisão de 1/10 de milímetro. Em sequiência foi introduzido através do colo do útero um cateter de duas vias (sonda Foley número 10), sendo uma com balonete inflável para obstrução do colo do útero e outra aberta para injeção de corante (azul de metileno) ou de ar sob pressão ( $80 \mathrm{mmHg}$ ) no interior da cavidade do útero.

Para o teste de pressão hidráulica a via aberta do cateter foi conectada a um dispositivo em Y. Num ramo do Y foi conectada uma seringa de $20 \mathrm{~mL}$ e no outro ramo foi conectado um manômetro. Pela seringa foi injetado ar atmosférico no sistema até atingir a marca de $80 \mathrm{mmHg}$. A peça operatória ficou imersa o tempo todo em cuba metálica contendo solução salina. O teste foi considerado positivo para perviedade se houvesse saída de bolhas de ar pelas fímbrias ou do local seccionado. Neste momento foi anotada a pressão responsável pelo avulsão do tampão de adesivo. Quando não ocorreu a saída de ar, até cinco minutos após a pressão máxima ser atingida e mantida (teste da pressão de rompimento), o teste foi considerado negativo para a perviedade.

Para o teste do corante foram injetados $20 \mathrm{~mL}$, sob pressão $(80 \mathrm{mmHg})$, de azul de metileno que ocupou toda a cavidade do útero e fez pressão sobre o istmo da tuba no local de inserção do adesivo. A presença de corante nas fímbrias das tubas uterinas, ou no local seccionado, foi considerando positivo e sinal de perviedade.

Os resultados obtidos foram submetidos aos testes estatísticos paramétricos não pareados (teste $t$ de Student) e para a porcentagem de ocorrências o teste de Fischer. O nível de significância foi menor ou igual a $5 \%$ para rejeição da hipótese de nulidade $(\alpha \leq 0,05)$. 


\section{RESULTADOS}

As três ovelhas do grupo Sham (G-SH) ficaram prenhas durante os noventa dias do acasalamento e foram poupadas da eutanásia levando à termo a gestação com prole saudável. As ovelhas submetidas à ligadura (G-PO) ou aplicação do n-butil-2-cianoacrilato (G-AD) não ficaram prenhas apesar de ficarem os noventa dias em presença dos mesmos machos que fecundaram as ovelhas do grupo $\mathrm{SH}$.

Nos animais do grupo PO percebeu-se macroscopicamente a presença de granulomas provocado pelo fio de sutura e de aderências peritoneais, em todos os animais (100\%), no local da ligadura. Não observamos a presença de granulomas e nem de aderências peritoneais na tuba uterina no grupo $\mathrm{AD}(0 \%)$, sendo o adesivo visível por transparência. A morfometria mostrou aumento estatisticamente significante no diâmetro da tuba uterina nos animais do G-AD $(0,650 \pm$ $0,16 \mathrm{~cm})$ em relação ao G-PO $(0,195 \pm 0,04 \mathrm{~cm}) *(\mathrm{p}=0,01 *)$, sendo o comprimento do implante de $0,45 \pm 0,05 \mathrm{~cm}$.

Os testes de perviedade mostraram que não houve passagem de corante (Figuras 1-A e B) ou do ar (Figura 1-Ce D) em nenhum dos animais do grupo PO. Nos animais do grupo adesivo todos foram negativos para o teste do corante, no entanto um dos lados foi positivo para o teste de pressão hidráulica.

\section{DISCUSSÃO}

A literatura refere que histeroscopia com obstrução da tuba uterina transcervical tem-se mostrado mais vantajosa em termos de custos e de intercorrências operatórias do que a laqueadura por via laparoscópica. No entanto, ainda é objeto de controvérsia qual a substância ou meio mecânico que se

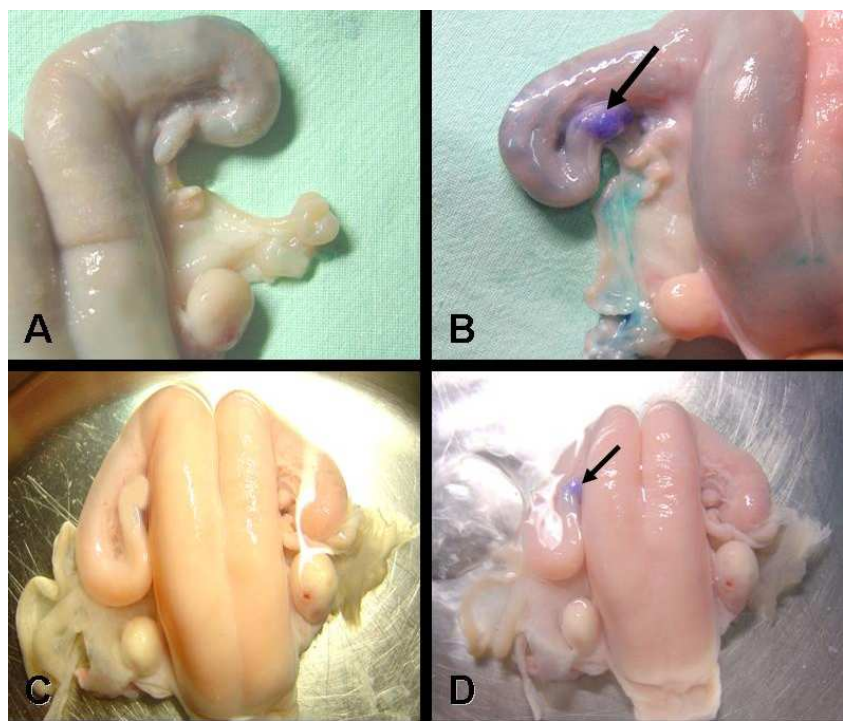

Figura 1 - Fotografias mostrando o teste de perviedade com uso do azul de metileno (A e B) e com pressão hidráulica $(C$ e $D)$ em tubas uterinas de ovelhas. Em (A) notar teste negativo mostrando a dilatação produzida pelo corante a montante da ligadura num animal do grupo Pomeroy $(P O)$, e em $(B)$ teste negativo num animal do grupo adesivo $(A D)$ Notar em $(B$ e $D)$ a presença, por transparência, do adesivo (setas). deva utilizar para obstruir o lúmen da tuba uterina ${ }^{4,14,15}$. As várias tentativas com o uso de implantes (silicone, hidrogel, microfusos metálicos), ablação do endométrio do corpo uterino (lasers, diodo ou radiofreqüência) ou aplicação de substâncias esclerosantes ou adesivos de fibrina não mostraram resultados consistentes para uma aplicação rotineira ${ }^{14,15}$.

Atualmente o Essure ${ }^{\circledR}$ é o único dispositivo aprovado pela FDA (Food and Drug Administration - USA) e pelo EHO (European Health Office) cujo estudo está na fase III de avaliação clínica multicêntrica ${ }^{16}$. Trata-se de dispositivo expansível ( $2 \mathrm{~mm}$ X $4 \mathrm{~cm})$, confeccionado em titânio e níquel envolvido por uma capa de dacron e tem como objetivo criar um processo inflamatório no local do implante com fibrose intramural da tuba ${ }^{4}$. Considerando que o dispositivo provoca fibrose em uma área extensa $(4 \mathrm{~cm})$ isto poderá ser um óbice para uma eventual recanalização, pois no Brasil a taxa de arrependimento da laqueadura gira em torno de $25 \%{ }^{1,2}$. Também há que se considerar o custo do dispositivo em si (US\$1.370,00) ${ }^{14}$.

A associação da histeroscopia e do adesivo de cianoacrilato pareceu uma opção viável, uma vez que o mesmo tem-se mostrado seguro e eficaz na obstrução de fístulas digestivas, urinárias e pulmonares ${ }^{5,6}$. Uma pesquisa sobre a esterilização transvaginal de coelhas com metil-cianoacrilato, após duas semanas de observação, mostrou a presença de necrose, células inflamatórias, fibrina e fibrose ${ }^{9}$. Após dois e seis meses ocorreu a total absorção do adesivo e todas as tubas mostraram fibrose intramural e processo inflamatório crônico ${ }^{9}$. Outra pesquisa refere a aplicação de $0,15 \mathrm{~mL}$ de metilcianoacrilato no lúmen tubário de coelhas, através de uma incisão no corno uterino dois centímetros distante da sua junção com a tuba. Após seis semanas todas as tubas estavam obstruídas, com intensa fibrose e ausência do adesivo. Partículas do polímero foram encontradas em macrófagos ${ }^{8}$. Por sua vez, só há uma referência na literatura sobre o uso do n-butil2-cianoacrilato. Duas mulheres foram submetidas a aplicação transvaginal do adesivo, guiado por fluoroscopia e após quatro anos de seguimento permaneciam com suas tubas obstruídas ${ }^{10}$. A histerossalpingografia foi o único meio de controle da eficácia do procedimento sem outras informações clínicas ou funcionais.

Considerando estes trabalhos passamos a investigar a aplicação do n-butil-2- cianoacrilato que possui maior capacidade de adesividade ${ }^{5,6}$. Em publicação anterior ${ }^{11}$ mostramos que a aplicação histeroscópica do n-butil-2-cianoacrilato em tubas uterinas de coelhas, em prazo curto de observação de trinta dias promoveu a obstrução total com mínimas alterações morfológicas na mucosa e no miossalpinge, sendo que o adesivo permaneceu íntegro no lúmen da tuba uterina. As coelhas, que já haviam tido ninhadas anteriores, cruzadas com machos comprovadamente férteis não ficaram novamente prenhes. O teste de perviedade por pressão hidráulica e o teste de corante mostraram total obstrução das tubas. Outro grupo de coelhas submetidas aos mesmos procedimentos, mas com o uso de cola biológica de fibrina não tiveram as tubas obstruídas e todas ficaram prenhas ${ }^{11}$. Resultados semelhantes foram obtidos com os mesmos procedimentos, porém com observação seriada de sessenta, noventa e, cento e oitenta $\operatorname{dias}^{12}$. 
Este estudo experimental de aplicação por meio de histeroscopia de n-butil-2-cianoacrilato no lúmen da tuba uterina em ovelhas observadas por noventa dias foi eficaz para obstruir o lúmen tubário e impedir a fecundação neste período, não provocou a formação de granulomas e nem de aderências, além de não ocorrer a absorção do adesivo. $\mathrm{O}$ teste de perviedade tubária, por instilação de corante, mostrou-se negativo, sendo que a macroscopia demonstrou a presença do n-butil-2-cianoacrilato circunscrito ao local da aplicação na luz da tuba uterina.

No grupo da laqueadura foram encontradas inúmeras aderências no local da cirurgia, em praticamente todos os animais. Deve ser ressaltado que as aderências após cirurgia abdominal estão associadas à inúmeras complicações como dor abdominal e pélvica crônicas, assim como obstrução intestinal $^{17}$.

O adesivo não deve ter provocado reação inflamatória importante, visto que à macroscopia não se evidenciou comprometimento da camada serosa e consequentemente ausência total de qualquer tipo de aderência peritoneal. O tampão de adesivo ocupou a extensão máxima de $0,5 \mathrm{~cm}$ e a área comprometida ao redor da aplicação não ultrapassou $0,2 \mathrm{~cm}, o$ que pode se considerar uma vantagem em relação ao Essure ${ }^{\circledR}$ que tem $4 \mathrm{~cm}$ de extensão e provoca fibrose intramural. $\mathrm{Na}$ eventualidade de uma recanalização este fato pode ser fator decisivo no êxito do procedimento.

A não absorção do adesivo é fato inusitado em relação ao seu uso na intimidade de outros tecidos onde comumente ele é reabsorvido. Pelo comportamento biológico do adesivo esperava-se uma reação inflamatória e uma fibrose intramural discreta. A persistência do adesivo no lúmen tubário e sua pobre reação tecidual, visto não haver granulomas, podem ser consideradas como vantagens em relação aos dispositivos referidos na literatura para o mesmo fim ${ }^{14,16}$.

Os resultados do grupo Sham $(\mathrm{G}-\mathrm{SH})$ mostraram que a manipulação provocada pela histeroscopia, cateterização da tuba uterina e injeção de substância inerte (soro fisiológico) não causaram obstáculo à fertilização das ovelhas. $\mathrm{O}$ achado reforça o fato conhecido do procedimento de histeroscopia ${ }^{16,18}$ e cateterização da tuba uterina ser seguro e confiável.

O grupo de ligadura cirúrgica tipo Pomeroy (G-PO) foi escolhido por ser reconhecido como de uso corrente ${ }^{19,20}$. A via de acesso laparotômica foi escolhida, pois os dados nacionais mostram que as laqueaduras são feitas preferencialmente no momento do parto ${ }^{1,3}$, no entanto, para o modelo proposto para esta pesquisa a via laparoscópica deveria ser a escolhida por ser a mais apropriada para a realização de laqueadura interpartal $^{16,17}$. De qualquer modo pôde-se perceber que o método foi eficaz e esteve associado, como é de conhecimento comum $^{19,20}$, à formação de granulomas e aderências peritoneais.

A presente pesquisa trouxe maiores subsídios ao procedimento histeroscópico de obstrução de tubas uterinas, e coloca o adesivo de n-butil-2-cianoacrilato como uma opção viável que deve ser mais explorada e a associação de ambos abre perspectivas de atender às necessidades individuais e sociais do país nos procedimentos operatórios de planejamento familiar.

\begin{abstract}
Background: To evaluate, through pregnancy and pressure burst tests, the effectiveness of sheep uterine tube sterilization through hysteroscopic insertion of cyanoacrylate adhesive. Methods: Adult sheep $(n=12)$, with one previous pregnancy, were distributed as follows: PO Group (n=3) submitted to laparotomy and Pomeroy surgical procedure of tubal obstruction, Group $\mathrm{SH}(n=3)$ submitted to hysteroscopy application of saline solution inside the tubal isthmus, and Group $A D(n=6)$ submitted to hysteroscopy application of $0.5 \mathrm{~mL}$ of $n$-2-butil-cyanoacrylate inside the tubal isthmus. They were allowed to copulate with fertile males during ninety days. The non pregnant sheep, at the 90 th day, were submitted to laparotomy, the uterine tubes were removed and their diameters analyzed. The uterine tubes were evaluated for patency with methylene blue dye injection. The overpressure burst test $(80 \mathrm{mmHg})$ was then applied through the uterine cervix. Data were evaluated by $t$ Student and Fisher test. Results: All sheep from groups PO and AD did not get pregnant (0\%) in contrast with sheep from group SH (100\%); the adhesive remained stable inside the tubal lumen. The diameter of the proximal tube in AD group $(0.650 \pm 0.16 \mathrm{~cm})$ was significantly wider than the PO group $(0.195 \pm 0.04 * \mathrm{~cm})(p=0.01)$. The overpressure burst and dye patency tests were negative for all animals from $P O$ group and only one animal in $A D$ group was positive for the pressure burst test. Conclusion: The hysteroscopic insertion of cyanoacrylate inside the tubal lumen of the sheep was effective to obstruct the uterine tube and to promote the sterilization, and would be an option for future applying in human beings.
\end{abstract}

Key words: Fallopian tubes; Hysteroscopy; Sterilization, reproductive; Tissue adhesives; Sheep.

\section{REFERÊNCIAS}

1. Minella LS. Aspectos positivos e negativos da esterilização tubária do ponto de vista de mulheres esterilizadas. Cad Saúde Pública. 1998;14(Suppl.1):S69-S79.

2. Vieira EM. O arrependimento após a esterilização feminina. Cad Saúde Pública. 1998;14(Suppl.1):S59-S68.

3. Berquó E, Cavenaghi S. Direitos reprodutivos de mulheres e homens face à nova legislação brasileira sobre esterilização voluntária. Cad Saúde Pública. 2003;19(Suppl.2):S441-S53.
4. Ubeda A, Labastida R, Dexeus S. Essure: a new device for hysteroscopic tubal sterilization in an outpatient setting. Fertil Steril. 2004;82(1):196-9.

5. Fagundes DJ, Taha MO, Montero EFS. Adesivo em cirurgia. In: Burihan E, editor. Condutas em cirurgia. São Paulo: Atheneu, 2001. p. 685-90.

6. Rivoire HC, Taha MO, Fagundes DJ. Adesivos Cirúrgicos: revisão e atualização. J Bras Med. 2002; 82(3):101-3.

7. Sonmez AS, Arub I, Dunn RC, Kaufman RH, Chuong CJ. Sterilization with fibrin sealant in a rabbit uterine horn model. Am J Obstet Gynecol. 1997; 177(3):573-8. 
8. Corfman PA, Richart RM, Taylor HC. Response of the rabbit oviduct to a tissue adhesive. Science. 1965;148(4):1348-50.

9. Berkey GS, Nelson R, Zuckerman AM, Dillehay D, Cope C. Sterilization with methyl cyanoacrylate-induced fallopian tube oclusion from a nonsurgical transvaginal approach in rabbits. JVRI. 1995;6(5):669-74.

10. Pelage JP, Herbreteau D, Paillon JG, Murray JM, Rymer R, Garance P. Selective salpingography and fallopian tubal occlusion with n-butyl-2-cyanoacrylate: report of two cases. Radiology. 1998; 207(3):809-12.

11. Rivoire HC, Fagundes DJ, Taha MO. A aplicação transvaginal de n-butil-2-cianoacrilato e o estudo da perviedade das tubas uterinas de coelhas. Rev Col Bras Cir. 2003; 30(5):337-43.

12. Rivoire HC. Efeitos a longo prazo da aplicação de adesivos cirúrgicos na perviedade de tubas uterinas de coelhas [dissertação]. São Paulo (SP): Universidade Federal de São Paulo; 2006.

13. Noronha PB, Borelli V, Barbosa J. Estudo morfológico dos componentes do pedículo ovariano de ovelha. Rev Inst Cienc Saúde. 2003;21(4):351-6.

14. Levie MD, Chudnoff SG. Office hysteroscopic sterilization compared with laparoscopic sterilization: A critical cost analysis. J Minim Invasive Gynecol. 2005; 12(4):318-22.

15. Cooper JMJ, Carignan CS, Cher D, Kerin JF. Microinsert nonincisional hysteroscopic sterilization. Obstet Gynecol. 2003;102(1):59-67.

16. Kerin JF, Carignan CS, Cher D. The safety and effectiveness of a new hysteroscopic method for permanent birth control: results of the first Essure pbc clinical study. Aust N Z J Obstet Gynaecol. 2001;41(4):364-70.
17. La Gamma A, Letoquart JP, Kunin N, Chaperon J, Mambrini A. Obstruction of the small intestine caused by bridles and adherences. Analysis of 157 operated cases. J Chir. 1994;131(67):279-84.

18. MacKay AP, Kieke BA Jr, Koonin LM, Beattie K. Tubal sterilization in the United States, 1994-1996. Fam Plann Perspect. 2001;33(4):161-5.

19. Kohaut BA, Musselman BL, Ramos LS, Kaunitz AM. Randomized trial to compare perioperative outcomes of Filshie clip vs. Pomeroy technique for postpartum and intraoperative cesarean tubal sterilization: a pilot study. Contraception. 2004; 69(4):267-70.

20. Robinson DC, Stewart SK, Reitan RE, Gist RS, Jones GN. Laparoscopic Pomeroy tubal ligation: a comparison with tubal cauterization in a teaching hospital. J Reprod Med. 2004;49(9):717-20.

Como citar este artigo:

Bigolin S, Fagundes DJ, Rivoire HC, Simões RS, Fagundes ATN, Simões MJ. A aplicação de adesivo de cianoacrilato por histeroscopia e os testes de perviedade na esterilização tubária de ovelhas. Rev Col Bras Cir. [periódico na Internet] 2008; 35(1). Disponível em URL: http://www.scielo.br/rcbc

Endereço para correspondência:

Sergio Bigolin

Rua Adolfo Hofstaetter, 49 - Loteamento Schimdt

99700.000 - Erechim - RS

Fone: (54) 3321.3564 - Fax: (54) 3321.4602

E-mail: bigolin@itake.net.br 